\title{
Editorial Note \\ Special Issue on Discrete Graphical Models in Biomedical Image Analysis
}

\author{
Ben Glocker ${ }^{\mathrm{a}}$, Nikos Paragions ${ }^{\mathrm{b}}$, Ramin Zabih $^{\mathrm{c}}$ \\ ${ }^{a}$ Biomedical Image Analysis Group, Imperial College London \\ ${ }^{b}$ Center for Visual Computing, University of Paris Saclay / Inria \\ ${ }^{c}$ Cornell NYC Tech / Weill Cornell Radiology
}

\section{Introduction}

Discrete graphical models (DGMs) have emerged as an immensely powerful tool for modeling complex relations and dependencies between input and output data in all types of algorithms, systems and processing pipelines. DGMs are ubiquitous in many biomedical image analysis and computer vision methods, either used as general holistic frameworks for the underlying methodology or to model particular components which are represented and solved best through graphical models. An overview of different models and applications of DGMs are presented in a recent survey by Wang et al. (2013). While the initial success of DGMs was mostly limited to low-level image processing and computer vision tasks where graph nodes play the role of individual pixel observations in terms of random variables and edges between nodes represent dependencies, these days DGMs are employed to model much higher level tasks in various problems. With the advances in efficient combinatorial optimization techniques such as graph cuts, linear programming and variants of belief propagation it is now feasible to perform inference in models with complex higher-order dependencies which often occur in biomedical settings. A recent comparative study (Kappes et al. (2015)) investigates the performance of various optimization techniques for different models. The availability of open source implementations has added to their popularity. Those discrete optimization methods combine excellent performance with great efficiency. So its not surprising that DGMs are now successfully used in a wide range of applications. This special issue tries to capture a snapshot 
of some of the currently most advanced methods and applications in which DGMs play an important role. By no means all current trends and advances could be included, however, we believe with the presented selection of nine excellent articles the potential of DGMs and related methods are well represented. In the following we provide a brief overview of those articles. The editors would like to thank all authors for their contributions and the many submissions to this special issue. The great interest in this issue once more reflects the success and popularity of DGMs. Many thanks to all anonymous reviewers who helped with the selection of the papers. We hope the reader will enjoy this issue and that it will serve as a reference of the state-of-the-art in discrete graphical models in biomedical image analysis.

\section{Overview of the Papers}

\section{Detection and Segmentation}

Arteta et al. tackle the problem of detecting overlapping cell structures in microscopy images. They model the detection and identification of individual cells in a tree-structured discrete graphical model which allows to pose detection as a labeling problem that can be solved using global optimization.

Karimaghaloo et al. focus on detection and segmentation of small pathologies, such as small lesions in multiple sclerosis MRI. They introduce a multilevel conditional random field approach and incorporate higher-order cliques for modelling the CRF energy which is invariant to rotations and local intensity distortions.

Both automatic and interactive neuron segmentation in electron microscopy

are tackled via an efficient conditional random field approach by Uzunbas et al. Automated segmentation is achieved via hierarchical merging trees using watersheds. Interactive refinement is based on uncertainties and an efficient tree-structured inference algorithm.

Rajchl et al. present their hierarchical max-flow segmentation framework which allows to combine intensity, spatial and topological information into the energy formulation. Learned, large-scale Gaussian mixture models form the basis of their model and inference is performed via a convex max-flow optimization algorithm.

\section{Tracking and Registration}

Uncertainty estimation within a supervoxel based belief propogation framework for nonrigid image registration is introduced by Heinrich et al. Their 
framework allows to determine direct, dense voxel-wise estimation of the displacement vectors without the need for a course-to-fine strategy.

The work by Dorfer et al. is aiming to recover the trajectories of cancer cells in phase contrast video-microscopy. The problem is formulated as a shortest path search in a graph where edges connect cell detections between adjacent frames, and nodes correspond to projections of cells.

Mirzaalian et al. propose a skin lesion tracking system which employs a graphical model to formulate lesion matching as a graph labeling problem. An adaptive learning algorithm is applied to set the parameters of the matching obective.

\section{Image Reconstruction}

The problem of image reconstruction of compressed sensing MRI is tackled by Lai et al. through the employment of a graph-based redundant wavelet transform. Reconstruction is then formulated as a shortest path in a graph where nodes correspond to transformed image patches.

A Markov random field approach is proposed by Lekadir et al. for the problem of dense reconstruction of 3D cardiac fiber orientations in diffusion tensor imaging. Statistical constraints are used to relate missing and known fibers, and a consistency term ensures continuity of the obtained 3D meshes.

\section{References}

Kappes, J. H., Andres, B., Hamprecht, F. A., Schnörr, C., Nowozin, S., Batra, D., Kim, S., Kausler, B. X., Kröger, T., Lellmann, J., Komodakis, N., Savchynskyy, B., Rother, C., 2015. A comparative study of modern inference techniques for structured discrete energy minimization problems. International Journal of Computer Vision 115 (2), 155-184.

URL http://dx.doi.org/10.1007/s11263-015-0809-x

Wang, C., Komodakis, N., Paragios, N., 2013. Markov random field modeling, inference \& learning in computer vision \& image understanding: A survey. Computer Vision and Image Understanding 117 (11), 1610 - 1627. URL http://www.sciencedirect.com/science/article/pii/ S1077314213001343 